\title{
Emphysematous Liver Abscess and Disseminated Hypervirulent Klebsiella pneumoniae Infection in a Patient from Southern India
}

\author{
Amiya R Nayak ${ }^{1} \odot$, Ramu Ramadoss ${ }^{\circledR}$, Venkateswaran Ramanathan ${ }^{3}$, Niveditha K Honnarudraiah $^{4}$ (อ)
}

\begin{abstract}
The pyogenic liver abscess is usually polymicrobial and is seen as a complication of biliary disease or peritonitis. Over the past three decades, monomicrobial liver abscess caused by Klebsiella pneumoniae is being increasingly reported from South East Asian countries like Taiwan and Korea. It is a community-acquired infection caused by a distinct strain, hypervirulent $K$. pneumoniae, different from classical strain related to the healthcare-associated Klebsiella infections. Diabetes mellitus is a significant risk factor. Reports of the disseminated infection due to hypervirulent Klebsiella are very few in Indian literature. We report the successful management of a diabetic patient with emphysematous liver abscess, brain abscess, and meningitis caused by hypervirulent K. pneumoniae infection.

Keywords: Brain abscess, India, Klebsiella, Liver abscess.

Indian Journal of Critical Care Medicine (2022): 10.5005/jp-journals-10071-24131
\end{abstract}

\section{INTRODUCTION}

Pyogenic liver abscess is usually polymicrobial and is often secondary to peritonitis or biliary tract disease. Monomicrobial liver abscess caused by Klebsiella pneumonia has increasingly being reported from the Asian population in the last two to three decades. K. pneumonia is a clinically important species implicated in community-acquired and healthcare-associated infections. ${ }^{1}$ Unlike the classical strain, which is often associated with nosocomial infection, the "hypervirulent" K. pneumoniae has emerged recently and reported predominantly from Southeast Asian countries but not from India.

The hypervirulent type is phenotypically and clinically distinct from the "classic" variant. This virulent strain causes a communityacquired pyogenic liver abscess and can disseminate to distant sites like the eyes, brain, and lungs. ${ }^{2}$ Unlike the classic variant, antibioticresistance is infrequent. Here, we report the successful management of a diabetic patient with $K$. pneumoniae associated emphysematous liver abscess complicated by meningitis and brain abscess.

\section{Case Description}

A 48-year-old gentleman, car driver, presented with myalgia for 10 days, fever and jaundice for 4 days, and altered sensorium for a day. He was a known diabetic but with poor compliance. He had consumed alcohol almost daily for the past 10 years. His vitals were stable. He was icteric with liver palpable $6 \mathrm{~cm}$ below the costal margin. He was drowsy.

Laboratory reports revealed direct hyperbilirubinemia (total bilirubin- $8.2 \mathrm{mg} / \mathrm{dL}$, direct bilirubin $-5.08 \mathrm{mg} / \mathrm{dL}$ ) with transaminitis (aspartate aminotransferase- $613 \mathrm{IU} / \mathrm{L}$ and alanine aminotransferase-441 IU/L). Neutrophilic leucocytosis was present. Blood sugar was $420 \mathrm{mg} / \mathrm{dL}$. His glycated hemoglobin was $11.2 \%$. Renal parameters were normal. The abdominal ultrasonography showed two hypoechoic lesions suggestive of liver abscesses, the largest measuring $7 \times 5 \mathrm{~cm}$. Contrastenhanced computerized tomography (CT) scan of the abdomen
${ }^{1-3}$ Department of Medicine, Jawaharlal Institute of Postgraduate Medical Education and Research, Puducherry, India

${ }^{4}$ Department of Radiology, Jawaharlal Institute of Postgraduate Medical Education and Research, Puducherry, India

Corresponding Author: Ramu Ramadoss, Department of Medicine, Jawaharlal Institute of Postgraduate Medical Education and Research, Puducherry, India, Phone: +91 9910952320, e-mail: ramadoss2912@ gmail.com

How to cite this article: Nayak AR, Ramadoss R, Ramanathan V, Honnarudraiah NK. Emphysematous Liver Abscess and Disseminated Hypervirulent Klebsiella pneumoniae Infection in a Patient from Southern India. Indian J Crit Care Med 2022;26(3):381-383.

Source of support: Nil

Conflict of interest: None

suggested emphysematous transformation of the abscess with multiple gas pockets (Fig. 1A). He was initiated on ceftazidime and metronidazole empirically and later switched to meropenem as there was no clinical improvement. Aspirate showed blood mixed pus. On the third day of hospitalization, he was intubated due to worsening mental status and shifted to the intensive care unit (ICU).

A hypodense lesion was noted near the left corpus striatum in the CT brain (Fig. 1B). The same lesion showed ring enhancement and was consistent with brain abscess in magnetic resonance imaging(MRI) of the brain. The cerebrospinal fluid (CSF) analysis showed $24960 / \mathrm{mm}^{3}$ cells with neutrophilic pleocytosis (neutrophils-95\%), protein-493 mg/dL, and glucose-11 mg/dL were compatible with acute bacterial meningitis, though the CSF culture was sterile. Both blood culture and the abscess grew K. pneumoniae. The colonies were hypermucoviscous, as evidenced by a positive string test which suggested hypervirulent Klebsiella. The organism was sensitive to ceftriaxone, amoxicillin-clavulanate, ciprofloxacin, gentamicin, and cotrimoxazole. Hence, the antibiotic 

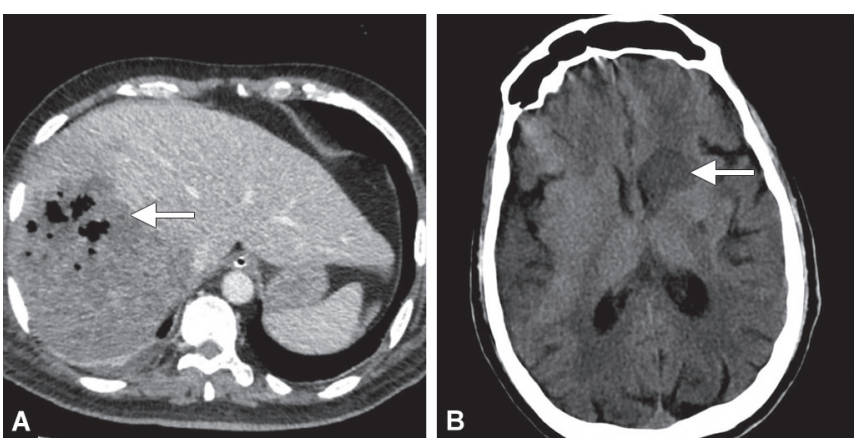

Figs $1 A$ and $B$ : (A) CT scan of the abdomen showing irregular hypodense lesion with multiple air foci in segment 5 and 8 of the right lobe; (B) CT scan of the brain showing a hypodense lesion in the region of the left caudate nucleus and anterior limb of the left internal capsule
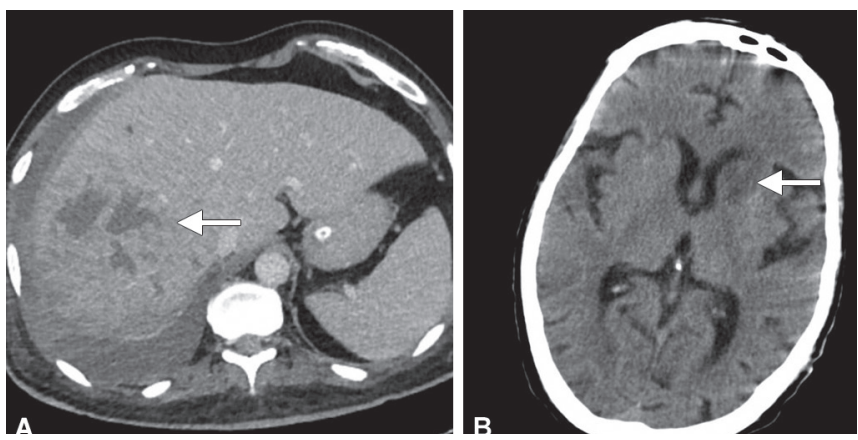

Figs 2A and B: (A) CT scan of the abdomen shows a decrease in the size of the abscess and disappearance of air pockets; (B) CT scan of the brain shows partial resolution of brain abscess

was de-escalated to ceftriaxone which was continued for 42 days. Liver function improved with treatment. We did a tracheostomy on the second week of ICU admission and subsequently weaned off the ventilator. Subsequent blood cultures were sterile. There was a significant radiological improvement in both brain and liver abscesses (Fig. 2). His neurological condition improved slowly. He was able to follow simple commands during discharge.

\section{Discussion}

While amoebic liver abscess continues to be the most common cause of community acquired liver abscess, with the changing epidemiology, one should be aware of Klebsiella as one of the emerging causes of community acquired liver abscess. Earlier reports on emphysematous liver abscess and disseminated amoebic abscess from India are available in literature. ${ }^{3,4}$ Reports from Taiwan and Korea about $K$. pneumoniae causing disseminated liver abscess are also available in literature. ${ }^{2}$ However, disseminated infection caused by hypervirulent variant has not been reported earlier from India.

The incidence of pyogenic liver abscess varies from 8 to 22 per million population. ${ }^{5}$ Ghosh et al. reported that $69 \%$ of liver abscess were due to Amoebic infection, and $18 \%$ were pyogenic in a study conducted in Delhi. ${ }^{6}$ Pyogenic liver abscess is usually polymicrobial. Another Indian study on pyogenic liver abscess shows that men are predominantly affected in their middle or old age, and K. pneumoniae was the leading aerobic organism grown in either in blood or pus aspirated from the liver abscess. ${ }^{7}$
Biliary tract diseases, peritonitis, and bowel infections usually precede pyogenic liver abscess. In contrast, Klebsiella associated liver abscess is mostly cryptogenic and has no underlying biliary or bowel diseases, as seen in our case. Invasive disease is associated with poorly controlled diabetes mellitus. Excessive alcohol consumption also increases the risk of liver abscess. ${ }^{8}$ Our patient had both of the risk factors, diabetes and alcoholism.

Emphysematous liver abscess accounts for $6-24 \%$ of the bacterial liver abscesses. ${ }^{9}$ K. pneumoniae was the causative organism for $84 \%$ of emphysematous liver abscess in a study. ${ }^{10}$ It shows a fulminant course with mortality rate of $27-30 \%$, as compared to $2-12 \%$ among patients with non-gas forming liver abscess. Drainage of abscess, appropriate antibiotics for 2-4 weeks, and strict glycemic control are the mainstays of management of pyogenic liver abscess. ${ }^{11}$ Patients should be considered for surgical intervention if the abscess is large, septate, multiple, or when percutaneous drainage fails. ${ }^{12}$

Brain abscess due to K. pneumoniae is less common, more debilitating, and seen with disseminated infection. Treatment requires antibiotics and surgical drainage if accessible. A prolonged antimicrobial course of 4-6 weeks is recommended..$^{13}$ In our case, K. pneumoniae was isolated from both emphysematous liver abscess and blood. Brain abscess was not aspirated. Although CSF analysis was suggestive of pyogenic meningitis, the culture was sterile, probably because antibiotics were started before CSF sampling.

K. pneumoniae strain causing invasive diseases like liver abscess produces a "hypermucoviscous" polysaccharide capsule. Due to this, extreme stickiness of colonies is noticed in the form of a positive string test. Among 78 different capsular serotypes, K1, less commonly K2, serotypes produce this hypermucoviscous capsule. ${ }^{14}$ The serotyping of the capsule of K. pneumoniae could not be done in our case. However, we firmly believe that it could be an infection of community-acquired hypervirulent (hypermucoviscous) strain because of its occurrence in a diabetic without any abdominal disease, dissemination, and a positive string test in the culture plate.

Although the hypervirulent strain of $K$. pneumoniae causes pyogenic liver abscess, it remains sensitive to commonly used antibiotics. So early detection and treatment will reduce the morbid sequelae and mortality.

\section{Conclusion}

Community-acquired pyogenic liver abscess due to $K$. pneumoniae is probably on the rise among Asian countries. Clinicians should be aware of the emerging hypervirulent strain of $K$. pneumoniae which can cause liver abscess and disseminated disease. Early diagnosis and percutaneous drainage are the critical steps in management. Hypervirulent strain is not usually resistant to common antibiotics.

\section{ORCID}

Amiya R Nayak @ https://orcid.org/0000-0002-3566-7484

Ramu Ramadoss (1) https://orcid.org/0000-0002-2913-4649

Venkateswaran Ramanathan (1) https://orcid.org/0000-0002-16025785

Niveditha K Honnarudraiah @ (1) https://orcid.org/0000-0002-64079972

\section{References}

1. Podschun R, Ullmann U. Klebsiella spp. as nosocomial pathogens: epidemiology, taxonomy, typing methods, and pathogenicity 
factors. Clin Microbiol Rev 1998;11(4):589-603. DOI: 10.1128/CMR. 11.4.589.

2. Jun JB. Klebsiella pneumoniae liver abscess. Infect Chemother 2018;50(3):210-218. DOI: 10.3947/ic.2018.50.3.210.

3. Shah MY, Vij AS, Shah F, Seth S, Pandit NA. Amoebic liver abscess with metastatic amoebic brain abscess: a case report. Ann Trop Med Public Health 2016;9(1):76-79. DOI: 10.4103/1755-6783.174724.

4. Kinsley JD, Nisheeth TP, Francis G, Srinivas CN. Aberrant form of Klebsiella pneumoniae in fulminant emphysematous hepatitis with polymicrobial infection. Era's Journal of Medical Research 2017;4(1):35-37. DOI: 10.24041/ejmr2017.6.

5. Serraino C, Elia C, Bracco C, Rinaldi G, Pomero F, Silvestri A, et al. Characteristics and management of pyogenic liver abscess: a European experience. Medicine (Baltimore) 2018;97(19):e0628. DOI: 10.1097/MD.0000000000010628.

6. Ghosh S, Sharma S, Gadpayle AK, Gupta HK, Mahajan RK, Sahoo R, et al. Clinical, laboratory, and management profile in patients of liver abscess from northern India. J Trop Med 2014;2014:142382. DOI: $10.1155 / 2014 / 142382$

7. Kumar SK, Perween N, Omar BJ, Kothari A, Satsangi AT, Jha MK, et al. Pyogenic liver abscess: clinical features and microbiological profiles in tertiary care center. J Family Med Prim Care 2020;9(8):4337-4342. DOI: 10.4103/jfmpc.jfmpc_927_20.

8. Liu Y, Wang JY, Jiang W. An increasing prominent disease of Kebsiella pneumoniae liver abscess: etiology, diagnosis, and treatment. Gastroenterol Res Pract 2013;2013:258514. DOI: 10.1155/ 2013/258514.

9. Lee HL, Lee HC, Guo HR, Ko WC, Chen KW. Clinical significance and mechanism of gas formation of pyogenic liver abscess due to Klebsiella pneumoniae. J Clin Microbiol 2004;42(6):2783-2785. DOI: 10.1128/JCM.42.6.2783-2785.2004.

10. Chou FF, Sheen-Chen SM, Chen YS, Lee TY. The comparison of clinical course and results of treatment between gas-forming and non-gas forming pyogenic liver abscess. Arch Surg 1995;130(4):401-405. DOI: 10.1001/archsurg.1995.01430040063012.

11. Takano Y, Hayashi M, Niiya F, Nakanishi T, Hanamura S, Asonuma K et al. Life-threatening emphysematous liver abscess associated with poorly controlled diabetes mellitus: a case report. BMC Res Notes 2017;10(1):117. DOI: 10.1186/s13104-017-2445-8

12. Heneghan HM, Healy NA, Martin ST, Ryan RS, Nolan N, Traynor O, et al. Modern management of pyogenic hepatic abscess: a case series and review of the literature. BMC Res Notes 2011;4:80. DOI: 10.1186/ 1756-0500-4-80.

13. Alvis Miranda H, Castellar-Leones SM, Elzain MA, Moscote-Salazar LR. Brain abscess: Current management. J Neurosci Rural Pract. 2013;4(Suppl 1): S67-S81. DOI: 10.4103/0976-3147.116472.

14. Doud MS, Grimes-Zeppegno R, Molina E, Miller N, Balachandar D, Schneper L, et al. A k2A-positive Klebsiella pneumoniae causes liver and brain abscess in a Saint Kitt's man. Int J Med Sci 2009;6(6): 301-304. DOI: 10.7150/ijms.6.301. 